\title{
An Unusual Complication of Pyogenic Liver Abscess
}

\author{
Hemanta K. Nayak, Kamlesh Kumar, Vivek A. Saraswat ${ }^{1}$, Gaurav Pandey ${ }^{3}$, Samir Mohindra ${ }^{2}$, Ankur Singh \\ Department of Gastroenterology, Sanjay Gandhi Postgraduate Institute of Medical Sciences, Lucknow 226014, India
}

\section{QUESTION}

A 27-year-old male was admitted with high-grade fever, right upper quadrant pain and malaise of 2 weeks duration. There was no history of diarrhoea, dysentery or vomiting. On examination, he was pale, toxic, febrile $\left(102{ }^{\circ} \mathrm{F}\right)$, with pulse 94 beats/min, blood pressure $110 /$ $80 \mathrm{mmHg}$ and respiratory rate 24 breaths/min.

The liver was palpable $2 \mathrm{~cm}$ below the right costal margin and was firm, tender and smooth. Examination of cardiovascular, respiratory and central nervous systems was unremarkable. Ultrasonography of abdomen (USG) revealed right lobe liver abscess with loculated right-sided pleural effusion. Laboratory examinations showed leucocytosis with normal serum bilirubin and serum creatinine. Blood culture was sterile and amoebic serology was negative.

Under local anaesthesia, a percutaneous drainage catheter (PCD) was placed into the abscess cavity and intravenous ceftriaxone ( $1 \mathrm{~g} \quad 12$ hourly) and metronidazole (intravenous $750 \mathrm{mg}$ thrice daily) were started. Four hundred millilitres of pus was drained on the table and $40-50 \mathrm{ml}$ of similar pus drained daily for next 8 days. However, the patient continued to be febrile over this period. In view of persistent symptoms, blood and drain culture were repeated and computed tomography (CT) of abdomen (Figure 1) and 2D echocardiography were requested (Figure 2). He was started on appropriate antibiotics based on culture report. His fever subsided and percutaneous drainage gradually decreased. Repeat echocardiography revealed resolution of previous lesions.

\section{DIAGNOSE THE ABOVE CLINICAL CONDITION}

Answer: Right atrium (RA) and inferior venacaval (IVC) thrombosis complicating pyogenic liver abscess

Pyogenic liver abscess may have various complications which includes rupture into the bile ducts or into pleural, pericardial, peritoneal cavities and vascular thrombosis. There are only a few case reports in which this complication

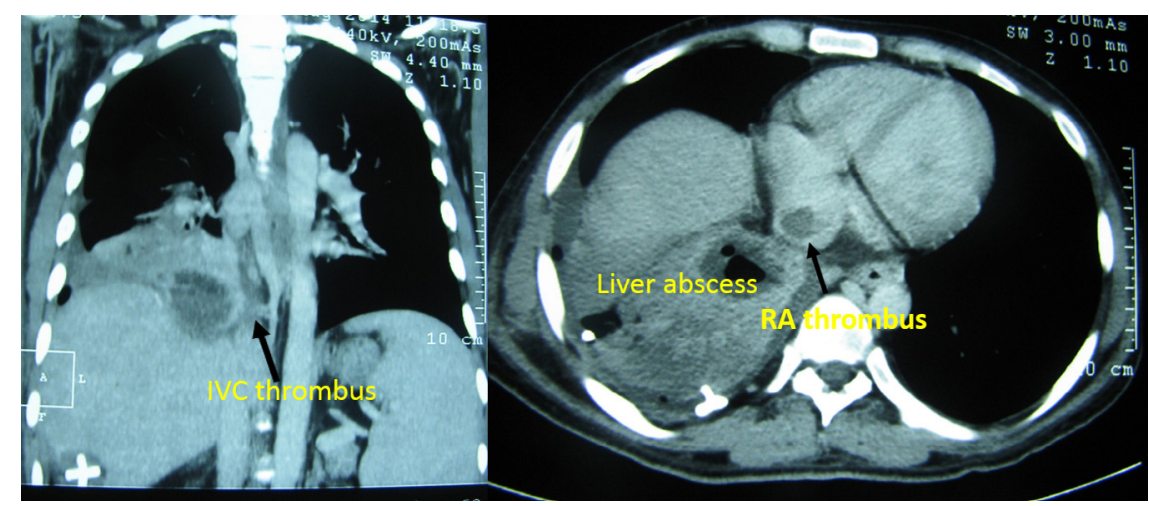

Figure 1 CT abdomen revealed right atrial and IVC thrombosis and abscess cavity extended close to the venous structures.

Keywords: pyogenic liver abcess, RA thrombosis, IVC thrombosis

Available online: 19 July 2016

Address for correspondence: Dr. Samir Mohindra, Additional Professor, Department of Gastroenterology, C Block, Sanjay Gandhi Post Graduate Institute of Medical Sciences, Lucknow 226014, India. Tel.: +91 8004904782.

E-mail: samir@sgpgi.ac.in

Abbreviations: RA: right atrium; IVC: inferior venacava; CT: computated tomography

http://dx.doi.org/10.1016/j.jceh.2016.07.004

${ }^{1}$ Professor and Head.

${ }^{2}$ Additional Professor.

${ }^{3}$ Assistant Professor. 


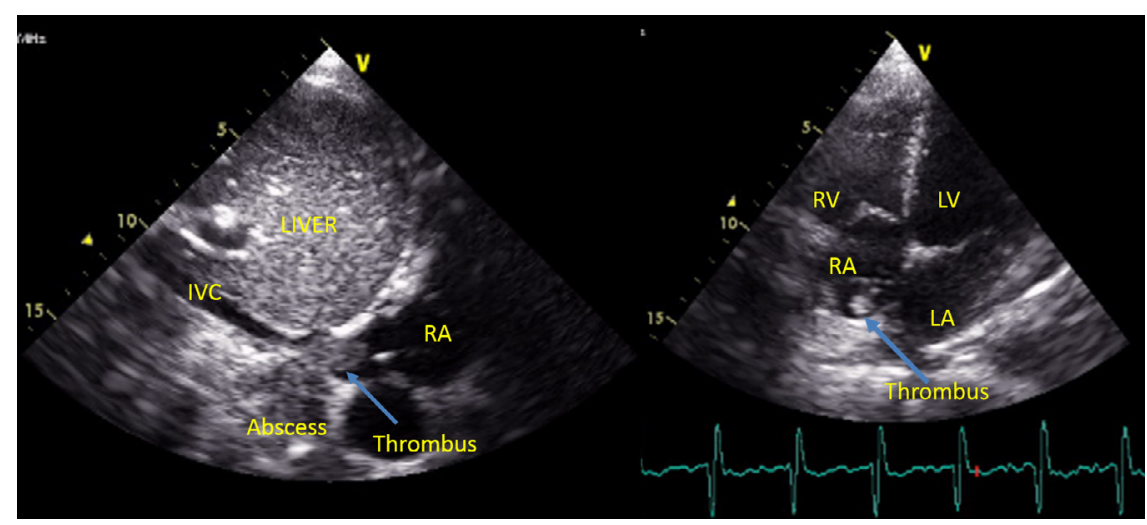

Figure 2 Echocardiography revealed a $24 \mathrm{~mm} \times 16 \mathrm{~mm}$ thrombotic mass extending into RA.

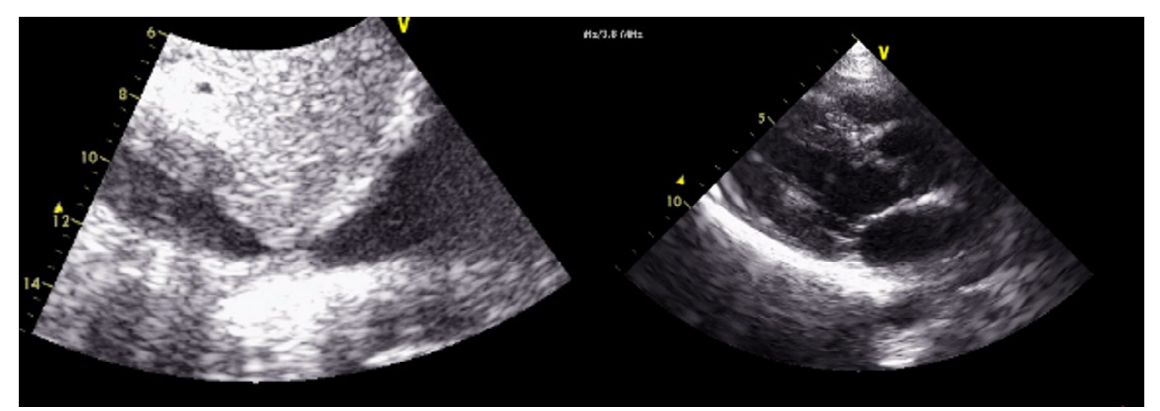

Figure 3 Repeat echocardiography revealed resolution of thrombus in IVC-RA junction.

of vascular thrombosis has been described. This patient had an unusual vascular complication with inferior vena caval thrombosis that extended into RA.

The presence of the atrial and IVC thrombosis was seen in CT scan (Figure 1A), and the liver abscess cavity extended close to the venous structures. Echocardiography revealed a $24 \mathrm{~mm} \times 16 \mathrm{~mm}$ thrombotic mass extending into RA (Figure 2A), and it is likely that the inflammatory process in the wall of the liver abscess spread directly to the adjacent wall of the IVC, inducing luminal thrombosis. The thrombus later propagated into the right atrium. The second blood culture revealed growth of Kleibsella pneumonia, which was resistant to previous antibiotics and sensitive to colistin and amikacin. He responded well to 4 weeks of colistin (intravenous $150 \mathrm{mg}$ twice daily) and amikacin (intravenous $750 \mathrm{mg}$ once daily) therapy. Repeat echocardiography revealed resolution of thrombus in IVC-RA junction (Figure 3).
The liver abscess and IVC thrombosis mostly managed by antibiotics and drainage of the abscess, but in a few cases, anticoagulation therapy may be needed to achieve complete resolution. ${ }^{1}$ Extension of thrombus up to right atrium may require aggressive management with thrombectomy to reduce the systemic complication. ${ }^{2}$

\section{CONFLICTS OF INTEREST}

The authors have none to declare.

\section{REFERENCES}

1. Sodhi KS, Ojili V, Sakhuja V, Khandelwal N. Hepatic and inferior vena caval thrombosis: vascular complication of amoebic liver abscess. J Emerg Med. 2008;34:155-157.

2. Hodkinson J, Couper-Smith J, Kew MC. Inferior vena caval and right atrial thrombosis complicating an amoebic hepatic abscess. Am J Gastroenterol. 1988;83:786-788. 